\title{
Using Moderate Transgene Expression to Improve the Genetic Sexing System of the Australian Sheep Blow Fly Lucilia cuprina
}

\author{
Ying Yan ${ }^{1, *(\mathbb{D})}$, Megan E. Williamson ${ }^{2}$ and Maxwell J. Scott ${ }^{2, *(\mathbb{C})}$ \\ 1 Department of Insect Biotechnology in Plant Protection, Institute for Insect Biotechnology, \\ Justus-Liebig-University Giessen, Winchesterstraße 2, 35394 Giessen, Germany \\ 2 Department of Entomology and Plant Pathology, North Carolina State University, Campus Box 7613, \\ Raleigh, NC 27695-7613, USA; mewilli9@ncsu.edu \\ * Correspondence: Ying.Yan@agrar.uni-giessen.de (Y.Y.); mjscott3@ncsu.edu (M.J.S.)
}

Received: 28 October 2020; Accepted: 11 November 2020; Published: 13 November 2020

Simple Summary: Populations of pest insects can be suppressed through repeated mass releases of sterilized insects. This is particularly effective if only sterile males are released. We previously developed several genetically modified strains of the Australian sheep blowfly that produce only males when raised on diet that lacked tetracycline. A disadvantage of the some of the engineered strains was that females would lay few eggs unless fed a diet with a low dose of tetracycline. In this study we show that effective male-only strains can be made by combining driver/effector lines that have moderate transgene expression/activity. Furthermore, the strain does not require tetracycline in the adult diet for female fertility. This "moderate expression/activity" strategy could be more generally applied to other pests that can be genetically modified.

Abstract: The sterile insect technique (SIT) is a promising strategy to control the Australian sheep blow fly Lucilia cuprina, a major pest of sheep. We have previously developed a transgenic embryonic sexing system (TESS) for this pest to facilitate the potential SIT application. TESS carry two transgenes, a tetracycline transactivator ( $t$ TA) driver and a tTA-activated pro-apoptotic effector. TESS females die at the embryonic stage unless tetracycline is supplied in the diet. However, undesired female sterility was observed in some TESS strains without tetracycline due to expression of tTA in ovaries. Here we investigate if TESS that combine transgenes with relatively low/moderate expression/activity improves the fertility of TESS females. tTA driver lines were evaluated for $t T A$ expression by quantitative real time PCR and/or by crossing with a tTA-activated RFPex effector line. Fertility and lethality tests showed that a TESS strain containing a driver line with moderate $t T A$ expression and an effector line showing moderate pro-apoptotic activity could recover the fertility of parental females and eliminated all female offspring at the embryonic stage. Consequently, such a strain could be further evaluated for an SIT program for L. cuprina, and such a "moderate strategy" could be considered for the TESS development in other pest species.

Keywords: livestock pest; Lucilia cuprina; genetic control; pro-apoptotic gene; sterile insect technique; genetic sexing; tetracycline-off system; insect transgenesis

\section{Introduction}

The Australian sheep blow fly Lucilia cuprina Wiedemann (Diptera: Calliphoridae) is a major livestock pest that poses a threat to the sheep industry in Australia, New Zealand and Africa [1-3]. Lucilia cuprina prefers warmer temperatures but could be expanding its range into historically cooler 
areas, presumably due to the changing climate [4]. Lucilia cuprina is a facultative parasite with females laying their eggs in an open wound or orifice and the developing larvae consuming the flesh of the host animal. Thus, in addition to causing considerable economic losses, L. cuprina is a major concern for animal welfare [5,6]. The sterile insect technique (SIT), which was successfully implemented to eradicate the New World screwworm Cochliomyia hominivorax Coquerel in North and Central America [7], was similarly proposed to battle against L. cuprina [8]. For the ongoing screwworm SIT program, insects are sterilized by radiation and both sexes are released into the field to mate with their wild counterparts. This approach is not optimal since sterile females and males that are released together can mate with each other, thus reducing the efficiency of suppression $[9,10]$. Indeed, large field cage tests demonstrated that male-only sterile releases of the Mediterranean fruit fly (Ceratitis capitata Wied.) increased the efficiency of population suppression by three-five-fold relative to bisexual releases [11].

To effectively remove females from the releasing population, a two-component genetic system was demonstrated in Drosophila melanogaster in which all females were eliminated at the pupal stage [12,13]. Subsequently, a transgenic embryonic sexing system (TESS) was developed for tephritid fruit flies including C. capitata and the Caribbean fruit fly Anastrepha suspensa [14,15]. We previously reported the generation of TESS strains for L. cuprina, which can eliminate all females at an early stage and lead to a male-only population $[16,17]$. TESS employs the tetracycline-off (Tet-off) system, which contains a "driver" component that expresses the tetracycline transactivator (tTA) gene under the control of a promoter mostly active in early stage embryos and a tTA-activated "effector" component that expresses a pro-apoptotic gene, L. sericata hid (Lshid). In the absence of tetracycline, tTA binds to the tet operator (tetO) sequences upstream of the Lshid exons and activates expression of Lshid in embryos at the cellular blastoderm stage. The Lshid effector gene contains the sex-specific first intron from the C. hominivorax transformer gene [18]. After RNA processing, only female transcripts encode functional LsHID protein due to the sex-specific RNA splicing. Consequently, all females die at either embryo [16] or early larval stage [17] depending on the promoter that controls the timing of $t T A$ expression during early development.

Ideally, the early promoter for $t T A$ regulation should only be active during embryogenesis and result in all females dying before the feeding stage. Since larval diet is a major cost in SIT programs, early female lethality would lead to significant savings in the rearing costs of the program. Typically, promoters from cellularization genes such as bottleneck, nullo and serendipity alpha are used to drive early embryo $t T A$ expression [14,15]. However, we have found that the promoters from the L. sericata bottleneck (Lsbnk) and spitting image genes (Lsspt) genes were also active at later stages of development including adults. Consequently, females from the TESS using these promoters were sterile and short-lived, possibly due to the LsHID production in the ovaries that was triggered by the "leaky" tTA expression $[16,17]$. Although female viability and fertility were restored by adding a high concentration of tetracycline to the diet until egg laying, such treatment resulted in any eggs laid carrying sufficient tetracycline to inhibit tTA in the developing embryo. Thus, it was necessary to find a feeding regimen of a low dose of tetracycline for only a few days after eclosion, which was sufficient for female fertility but would not prevent the female embryo lethal system from engaging in the next generation.

The activities of both driver and effector transgenes were impacted by negative genomic position effects, as is commonly associated with the piggyBac-mediated transformation system [16,19]. Indeed, some combinations of driver and effector lines showed high female survival in TESS from different species $[14,15,17,20]$. We considered the possibility that an effective TESS could be made by combining an effector line with moderate activity with a driver line that had low expression in adult females due to position effects. Here we have characterized several driver lines by quantitative real time (qRT)-PCR as well as by crossing with a tTA-inducible tetO-RFPex reporter line [21]. Multiple TESS strains were assembled using different driver and effector lines $[16,17]$. Fertility and lethality tests were performed to assess the performance of these TESS strains. We show that by combining "moderate" 
driver and effector lines an effective TESS strain can be made that does not require tetracycline in the adult diet.

\section{Materials and Methods}

\subsection{Fly Rearing and Germ-Line Transformation}

The LA07 wild type strain of L. cuprina was maintained and transformed as previously described [16]. Specifically, embryos were microinjected with a mixture of synthesized piggyBac RNA helper $(300 \mu \mathrm{g} / \mathrm{mL})$, Lchsp83-pBac helper $(200 \mu \mathrm{g} / \mathrm{mL})$ and $p B a c[$ Effector- $r p r]$ plasmid $(700 \mu \mathrm{g} / \mathrm{mL})$. First instar larvae showing transient expression of the DsRedex2 marker were selected and raised on raw $93 \%$ ground beef. $G_{0}$ adults were crossed to wild type flies and offspring screened for expression of the fluorescent marker at late embryo/first instar stages. Homozygous individuals were selected at the wandering third instar larval stage based on fluorescence intensity and bred to create a stable line.

\subsection{Plasmid Construction}

An Lsrpr fragment including 5'UTR and $3^{\prime}$ UTR [1] was amplified from L. sericata embryo cDNA using primers Ls-rpr-5UTR-F: 5'-GAGTTTCCATCTAGCAAACAAACAAT-3' and Ls-rpr-5UTR-R: 5'-TTATTTTTTAGCGGGTTTCACTTTT-3' , and cloned into pGEM-T (Promega) to form pGEM-Lsrpr. The Chtra intron fragment was amplified from pBS-FL3 [2] using primers NSWtra-StuI: 5'-TTTTAGGCCTCTAATTTTTTGAGCAACATT-3' and Lsrpr-NSWtra: 5'-CGGGTATATAGAATGCTACAGCCTAAACATAGAAAAGAATAATAAATTTATCATACA-3', and an Lsrpr fragment was amplified from pGEM-Lsrpr using primers NWStra-Lsrpr: 5'TGTATGATAAATTTATTATTCTTTTCTATGTTTAGGCTGTAGCATTCTATATACCCG-3' ${ }^{\prime}$ and LsrprTAA-BgIII: $\quad$ 5'-TCTGAGATCTTTATTTTTTAGCGGGTTTCACTTTTTTGTTG- ${ }^{\prime}$. The two PCR products were purified and combined as template for a second round of PCR, from which a Chtra_intron-ATG-Lsrpr fragment was amplified using primers NWStra-StuI and LsrprTAA-BgIII. Then the amplified fragment was ligated to pBS-FL3 [3] using unique StuI and BgIII sites to generate pBS-tetO21-Dmhsp70-Chtra_intron-ATG-Lsrpr-SV40 (pBS-effector-rpr). To replace the Dmhsp70 core promoter with the Lchsp70 core promoter in the plasmid pBS-effector-rpr, an Lchsp70-promoter-Chtra_intron fragment was cut from pBS-FL11 [3] and inserted into pBS-effector-rpr using unique BamHI and StuI sites. Finally, the tetO21-Lchsp70-Chtra_intron-ATG-Lsrpr-SV40 cassette was excised by digestion with XhoI and NotI and cloned into the unique XhoI and PspOMI sites in the piggyBac transformation vector pB[Lchsp83-DsRedex2] [4].

\subsection{RNA Isolation and $q R T-P C R$ Analysis}

Samples from different developmental stages and sexes were collected as before [16,17]. Total RNA was extracted using the RNeasy ${ }^{\circledR}$ Mini Kit (QIAGEN) according to the manufacturer's instructions. Isolated RNA was subsequently treated with the RNase-Free DNase Set (Qiagen). Then, $5 \mu \mathrm{g}$ RNA was used to synthesize cDNA using Superscript III First Strand Synthesis Supermix (Invitrogen) following the manufacturer's instructions. A control reaction lacking Superscript III was included for each preparation. qRT-PCR was performed as previously described with $L c G S T 1$ serving as the reference gene $[16,17]$.

The $2^{-\Delta \Delta C t}$ method was used with all samples relative to the $t T A$ expression in 2 days old females. The primers for the reference gene are LcGST1-F-GCCAGTGTCAGCACCTTTG and LcGST1-R-GCAACCTTCCCAGTTTTCATC. The primers for DR2 and DH1 strains are tTAo-F-TGTTGAATGAAGTGGGTATTGAAGGATTGACTACTCG and tTAo-R-CCAAAGGGCAAAAGTGGGTGTGATGTCTATC. The primers for DH4 strain are tTAV-F-TCTTGCGTAATAATGCCAAATCCTTCCG and tTAV-R-CCAACACACAGCCCAATGTAAAATGACC. 


\subsection{Confocal Imaging to Assess Ovary-Specific Expression of tTA}

The confocal images were acquired as previously described [21]. Briefly, homozygous males from one of the driver lines were crossed with homozygous virgin females from a tetO-RFPex reporter line. Ovaries were dissected from female offspring at four and eight days following eclosion and fixed by submerging in $4 \%$ paraformaldehyde (Biotium). Images were captured on a Zeiss LSM 880 confocal microscope using a 40× lens and the Argon and 561 lasers to capture green and red fluorescence, respectively. Additionally, all developmental stages were screened for red fluorescence using a Leica M205 FA Fluorescent Microscope.

\subsection{Female Lethality Test and Embryo-Specific Lethality Assessments}

Double homozygous (DH) strains which are homozygous for both the driver and effector transgenes, were generated using diet supplemented with $100 \mu \mathrm{g} / \mathrm{mL}$ tetracycline as previously described [16]. Specifically, homozygous virgin females from the effector lines were crossed with homozygous males from driver lines to generate double heterozygous strains. The double heterozygous strains were inbred and their progeny screened to select only individuals homozygous for both the driver and effector by epifluorescence microscopy based on fluorescence intensity. For female lethality tests, 10 pairs of newly emerged adults from a DH strain were used for each rearing bottle. Flies in these bottles were supplied with either $100 \mu \mathrm{g} / \mathrm{mL}$ tetracycline water for 8 days $(+\mathrm{W})$, tetracycline-free water $(-W)$ for 8 days or tetracycline water at a low concentration for two days then switched to tetracycline-free water for 6 days $(+-W)$. Embryos were then collected from each bottle and larvae were reared on either tetracycline-free meat $(-\mathrm{M})$ or meat containing $100 \mu \mathrm{g} / \mathrm{g}$ tetracycline $(+\mathrm{M})$. For staged lethality tests, 1000 embryos from the first egg lays of each DH strain were collected and reared on the tetracycline feeding regimen indicated. The number of 1 st instar larvae, 3rd instar larvae, pupae, and adult males and females were monitored.

\subsection{Statistical Analysis}

All statistics were carried out using SigmaPlot v14 (Systat Software). The differences in the $t$ TA expression level or number of flies from multiple sources were tested by one-way analysis of variance (ANOVA) and means were separated using the Holm-Sidak method. The number of flies from two sources were analyzed using two-sample $t$-tests as were the mean values from the number of male and female offspring from female lethality tests.

\section{Results}

\subsection{A Moderate Driver Line with Reduced Expression in Ovaries}

Previously reported TESS were made by combining a Lsbnk-tTA driver (DR2) or Lsspt-tTA driver (DR3) line with a tTA-activated Lshid effector (EF1 or EF3) [16,17]. Effector one (EF1) lines had a mutant version of Lshid, Lshid ${ }^{\text {Ala2 }}$, that was predicted to be resistant to inhibition by MAP kinase since the two conserved predicted MAPK phosphorylation sites (PPSP and PPTP) were mutated to encode Alanine rather than Serine or Threonine [22,23]. EF3 lines carried a wild type version of Lshid. Three DR2 lines $(\# 6, \# 7, \# 8)$ were analyzed here for $t$ TA expression in early embryos and young adult females using qRT-PCR. DR2\#7 had the lowest expression levels and DR2\#8 the highest (Figure 1a). Specifically, $t T A$ expression in early embryos (2 h old) and young females (2 days old) of DR2\#7 were 2.1 ( $p=0.440$, One-way ANOVA) and 2.0 ( $p=0.837$, One-way ANOVA) times lower than these in DR2\#6, and 2.7 ( $p=0.067$, One-way ANOVA) and 2.6 ( $p=0.623$, One-way ANOVA) times lower than in DR2\#8, respectively (Figure 1a). In addition, $t T A$ expression levels at different developmental stages were measured in two previously assembled TESS strains [16,17] (Figure 1b). In these strains, DR2\#6 was combined with the effector EF3E and DR3\#4 with the EF1-12 effector line. Both TESS were effective producing only males when raised on diet that lacked tetracycline. However, females produced very 
few eggs if tetracycline was not included in the adult diet $[16,17]$. The DR3\#4;EF1-12 strain showed higher $t$ TA levels than in the DR2\#6;EF3E strain at all stages examined (Figure 1b).

(a)

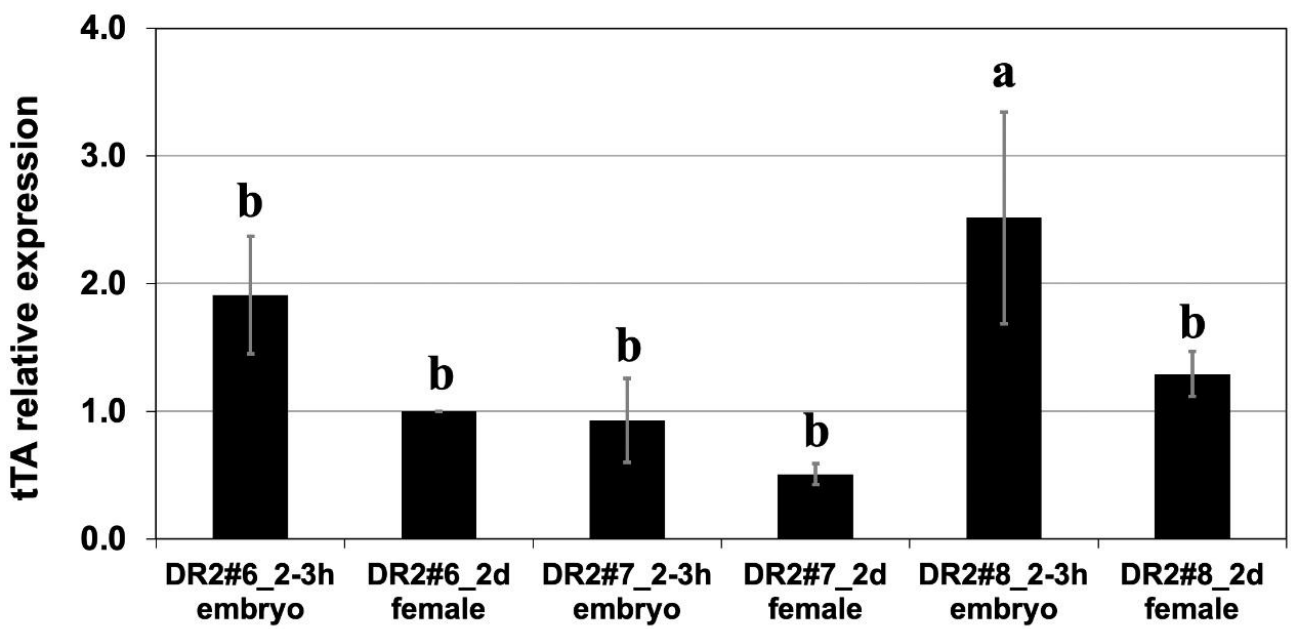

(b)

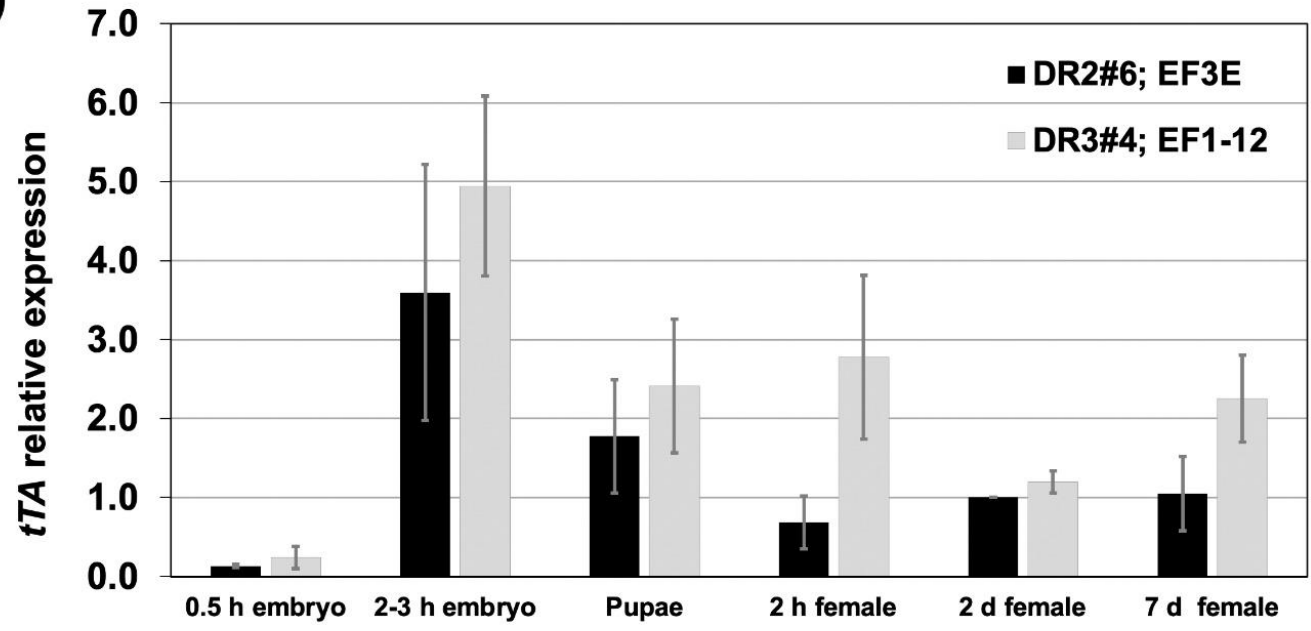

Figure 1. Evaluation of $t T A$ expression from different driver lines by qRT-PCR. (a). Relative $t T A$ expression levels in early embryo ( $2 \mathrm{~h}$ or $\mathrm{h}$ after egg laying) and young females ( 2 days or $\mathrm{d}$ after eclosion) of three DR2 driver lines were determined. The numbers with different lower-case letters are significantly different ( $p<0.05$, One-way ANOVA). (b) Relative tTA expression levels at different development stages of DH strains DR2\#6; EF3E and DR3\#4; EF1-12 are shown. The embryo samples were collected within $0.5 \mathrm{~h}$ or 2-3 h after egg laying and the female samples were collected $2 \mathrm{~h}, 2 \mathrm{~d}$ or $7 \mathrm{~d}$ after eclosion. For all qRT-PCR analysis, RNA levels are relative to the LcGST1 reference gene and data are from three biological replicates.

We recently verified that the DR2\#6 and DR3\#2 driver lines express tTA in ovaries through crossing with a tetO-RFPex reporter line [21]. Thus, the undesired sterility of females from the previous TESS that used these drivers was likely due to the activation of the strong pro-apoptotic Lshid gene in the ovaries (Figure 2a). We hypothesized that an effective TESS could be made by combining a driver line that had low expression in adult females with an effector line that had low basal activity due to negative position effects (Figure 2a). To evaluate such a "moderate strategy", DR2\#7, which showed the lowest $t T A$ expression in adult females (Figure 1a), was crossed with the tetO-RFPex line to evaluate if DR2\#7 would trigger the expression of the red fluorescent protein gene in ovaries. Red fluorescence was readily observed in the germarium of 4- and 8-day-old female offspring of a cross of the DR3\#4 driver with the tetO-RFPex line (Figure 2b, panels b1 and b2). Very weak fluorescence was observed 
in the germarium region of ovaries from 4-day-old females, and no red fluorescence was observed in ovaries from 8-day-old female offspring of a cross of the DR2\#7 driver with the tetO-RFPex line (Figure 2b, panels b3 and b4). Red fluorescence was observed in the embryos from a cross with either driver and tetO-RFPex (data not shown), confirming $t T A$ expression at the early stage. In addition, red fluorescence was observed in the $1^{\text {st }}$ and $3^{\text {rd }}$ instar larvae from the DR3\#4 cross, but not at these stages from the DR2\#7 cross (data not shown). The red fluorescence observed in DR3\#4;tetO-RFPex ovaries supports our earlier conclusion that DR3\#4;EF1-12 females were likely sterile because of activation of the Lshid gene by tTA [17]. Meanwhile, the very low red fluorescence in the ovaries of DR2\#7;tetO-RFPex females suggested that the DR2\#7 line could be used to make an effective TESS as part of the "moderate" strategy (Figure 2a).

(a)

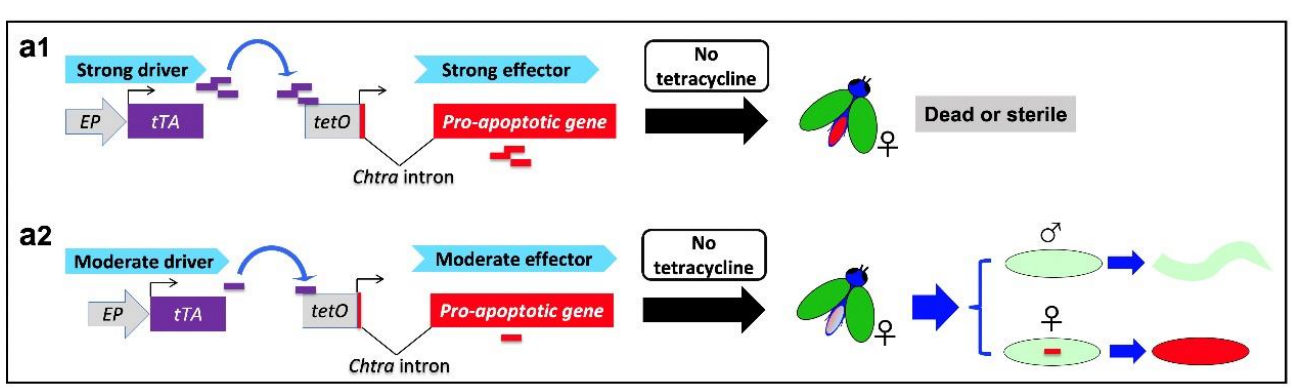

(b)
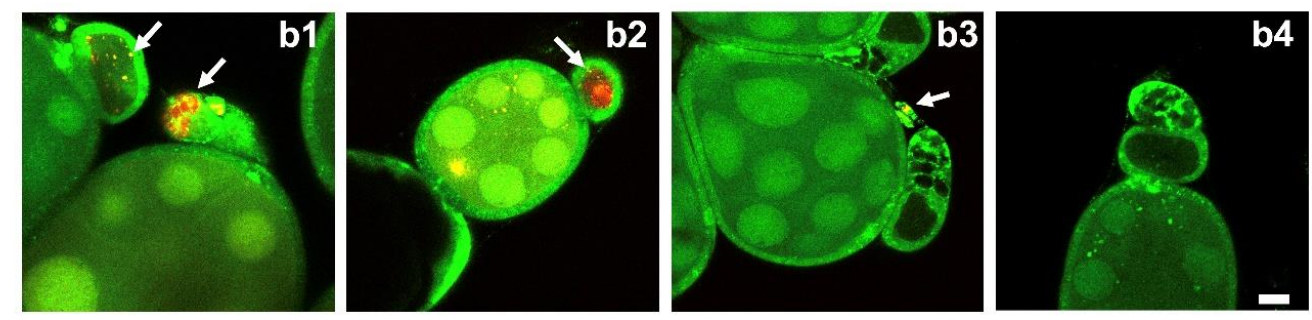

Figure 2. "Moderate driver and effector" strategy to improve the transgenic embryonic sexing system (TESS) of L. cuprina. (a) Schematic illustration for the transgenic embryonic sexing system (TESS) containing driver and effector components. The driver used an early promoter (EP) to drive the tetracycline transactivator ( $t T A$ ), and the effector contains a sex-specific intron (Chtra which immediately follows the ATG translation start codon of pro-apoptotic gene) splicing cassette within a pro-apoptotic gene to achieve highly efficient female lethality early in the development of L. cuprina. Unless adults are fed diet with tetracycline, females are sterile and short-lived if the early promoter is also active in female ovaries and the effector is strongly activated by tTA (a1) $[16,17,21]$. However, a combination of moderate driver and effector could reduce such "leaky" cell death in females thus an additional tetracycline supply may not be necessary (a2). (b) Confocal images of fluorescent ovaries. The images were taken using dissected ovaries from $4 \mathrm{~d}(\mathrm{~b} 1, \mathrm{~b} 3)$ and $8 \mathrm{~d}(\mathrm{~b} 2, \mathrm{~b} 4)$ old females, which were generated by crossing homozygous males from DR3\#4 (b1, b2) or DR2\#7 (b3, b4) with homozygous virgin females from EF-RFPex [21]. DsRed expression (indicated by white arrows) was observed in the early portion of the developing eggs (germarium). The nurse cells showed green fluorescence from the marker. Scale bar: $20 \mu \mathrm{M}$.

\subsection{The Combination of Selected Driver and Effector Lines Determines Female Fertility and Lethality}

Multiple strains were assembled by combining different driver and effector lines that had varying levels of expression or activity (Table 1). For effectors, we selected the lines EF3F, EF3A and EF1-13, which exhibited low, moderate and strong female-specific lethality, respectively, when previously crossed to DR2\#6 [16]. For drivers, we selected the lines DR2\#7 and DR2\#8, as tTA is expressed almost three times higher in line \#8 than \#7 (Figure 1a). Strains were bred to homozygosity for both driver and effector transgenes. Lethality tests were performed without tetracycline in the larval diet and with or without tetracycline added to the diet of the parental generation. The results show that the DH strains with the weak EF3F effector were fully viable and fertile without any tetracycline in 
the adult diet; however, female survival was high with either DR2\#7 or DR2\#8 (Table 1). With the moderate EF3A effector, if tetracycline was omitted from the adult diet, DR2\#7; EF3A females were fertile while DR2\#8; EF3A were sterile. Addition of 1 or $3 \mu \mathrm{g} / \mathrm{mL}$ tetracycline to the adult diet for the first two days after eclosion slightly increased pupal and male production of DR2\#7; EF3A. However, it was necessary to supply DR2\#8; EF3A females with a diet containing $50 \mu \mathrm{g} / \mathrm{mL}$ tetracycline to completely rescue female fertility (Table 1). The combination of DR2\#8 with EF1-13 required the adult diet be supplemented with $100 \mu \mathrm{g} / \mathrm{mL}$ tetracycline to restore pupal production. Nevertheless, any tetracycline passed on from DR2\#7; EF3A or DR2\#8; EF1-13 females was insufficient to rescue any female offspring that were reared on diet without tetracycline (Table 1). In addition, one tetO-Lsrpr effector line (EF4) was generated by piggyBac-mediated transformation and then combined with DR2\#6 or DR3\#4 driver lines. Adult females from these strains were viable and fertile without any tetracycline supply; however, all or most of females survived in the next generation (Figure S1). Specifically, in the absence of tetracycline, the DR2\#6; EF4 strain produced similar numbers of male and female offspring $(p=0.134, \mathrm{t}=1.877$, d.f. $=4)$, while the DR3\#4; EF4 strain produced significantly more males than females $(p=0.013, \mathrm{t}=4.298$, d.f. $=4)$ (Figure S1).

Table 1. Female fertility and lethality tests for different double homozygous strains.

\begin{tabular}{|c|c|c|c|c|c|c|}
\hline Strains & Tet Water ${ }^{a}$ & $\begin{array}{c}\text { Egg } \\
\text { Clutches }\end{array}$ & \#Pupae ${ }^{c}$ & \#Male & \#Female & Female (\%) \\
\hline Wildtype & 0 & $4-8$ & $843 \pm 105$ & $402 \pm 46$ & $376 \pm 33$ & 48.3 \\
\hline DR2\#7; EF3F & 0 & $4-8$ & $503 \pm 46$ & $248 \pm 28$ & $160 \pm 18$ & 39.2 \\
\hline $\begin{array}{l}\text { DR2\#7; } \\
\text { EF3A }\end{array}$ & 0 & $4-8$ & $143 \pm 28$ & $112 \pm 25$ & 0 & 0 \\
\hline $\begin{array}{l}\text { DR2\#7; } \\
\text { EF3A }\end{array}$ & 1 & $4-8$ & 193 & 159 & 0 & 0 \\
\hline $\begin{array}{l}\text { DR2\#7; } \\
\text { EF3A }\end{array}$ & 3 & $4-8$ & 197 & 153 & 0 & 0 \\
\hline DR2\#8; EF3F & 0 & $4-8$ & $359 \pm 38$ & $176 \pm 42$ & $101 \pm 11$ & 36.3 \\
\hline $\begin{array}{l}\text { DR2\#8; } \\
\text { EF3A }\end{array}$ & 0 & No eggs & & & & \\
\hline $\begin{array}{l}\text { DR2\#8; } \\
\text { EF3A }\end{array}$ & 1 & 1 & 5 & 3 & 0 & 0 \\
\hline $\begin{array}{l}\text { DR2\#8; } \\
\text { EF3A }\end{array}$ & 3 & $1-3$ & $41 \pm 7$ & $31 \pm 2$ & 0 & 0 \\
\hline $\begin{array}{l}\text { DR2\#8; } \\
\text { EF3A }\end{array}$ & 50 & $4-8$ & $249 \pm 14$ & $197 \pm 9$ & 0 & 0 \\
\hline $\begin{array}{l}\text { DR2\#8; } \\
\text { EF1-13 }\end{array}$ & 1 & No eggs & & & & \\
\hline $\begin{array}{l}\text { DR2\#8; } \\
\text { EF1-13 }\end{array}$ & 3 & No eggs & & & & \\
\hline $\begin{array}{l}\text { DR2\#8; } \\
\text { EF1-13 }\end{array}$ & 10 & No eggs & & & & \\
\hline $\begin{array}{l}\text { DR2\#8; } \\
\text { EF1-13 }\end{array}$ & 20 & No eggs & & & & \\
\hline $\begin{array}{l}\text { DR2\#8; } \\
\text { EF1-13 }\end{array}$ & 100 & $1-3$ & 321 & 296 & 0 & 0 \\
\hline
\end{tabular}

a Tetracycline at the indicated concentration $(\mu \mathrm{g} / \mathrm{mL})$ was supplied in the first two days after adult eclosion. $\mathrm{b}$ Tetracycline-free raw ground beef was used to collect eggs during a $24 \mathrm{~h}$ period on the 8 th day after eclosion and the number of egg clutches were counted. ${ }^{c}$ Data shown as mean \pm standard deviation for three biological replicates; Otherwise the data was from one biological replicate.

\subsection{Female Embryo-Specific Lethality of DH Strains with EF3A}

The DR2\#7; EF3A and DR2\#8; EF3A DH strains were further evaluated under different tetracycline feeding schemes. Both strains produced similar numbers of males and females on diet with $100 \mu \mathrm{g} / \mathrm{mL}$ tetracycline ( $p=0.189$, One-way ANOVA; Figure 3, $+\mathrm{W},+\mathrm{M}$ ). Most females died if the parental generation was fed a high dose of tetracycline $(100 \mu \mathrm{g} / \mathrm{mL})$ but the larval diet lacked tetracycline (Figure 3, $+\mathrm{W},-\mathrm{M})$, suggesting that the maternal tetracycline could rescue some female offspring. For DR2\#7; EF3A, a maternal supply of tetracycline was essential for female survival as none were 
rescued by adding tetracycline to the larval diet (Figure $3 a-W,+M)$, indicating all females died before the feeding stage. Importantly, DR2\#7; EF3A females were viable and fertile without any tetracycline supply, and produced only male offspring (Figure 3a-W, -M). However, DR2\#7; EF3A was not as productive as DR2\#7; EF3F or wild type, producing less than a third of the number of pupae from a similar number of egg clutches (Table 1). For DR2\#8; EF3A, females produced few offspring with a low dose of tetracycline $(3 \mu \mathrm{g} / \mathrm{mL})$, while a high dose of tetracycline $(50 \mu \mathrm{g} / \mathrm{mL})$ was needed to restore female fertility while also producing only male offspring in the next generation (Figure $3 b,+-W$, $+\mathrm{M})$. Staged lethality tests were carried out for strain DR2\#7; EF3A to further assess when females die (Figure 4). Compared to the number of first instar larvae that emerged from 1000 embryos from the constant tetracycline condition $(+\mathrm{W},+\mathrm{M})$, only about half the amount were found on diet without tetracycline $(-\mathrm{W},-\mathrm{M})$ or tetracycline only for the larval diet $(-\mathrm{W},+\mathrm{M})$. This result suggests females died at the embryo stage.

(a)

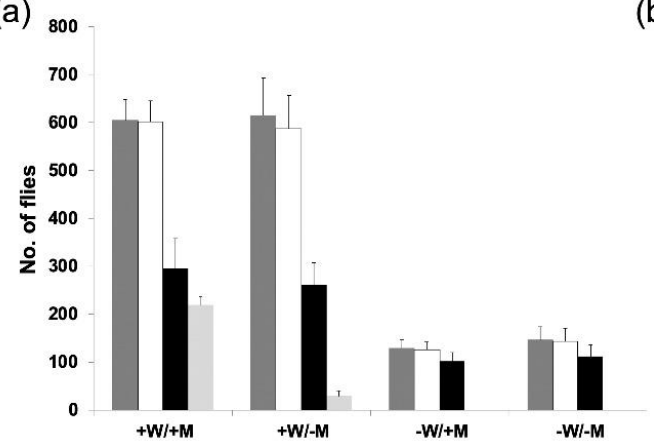

(b)

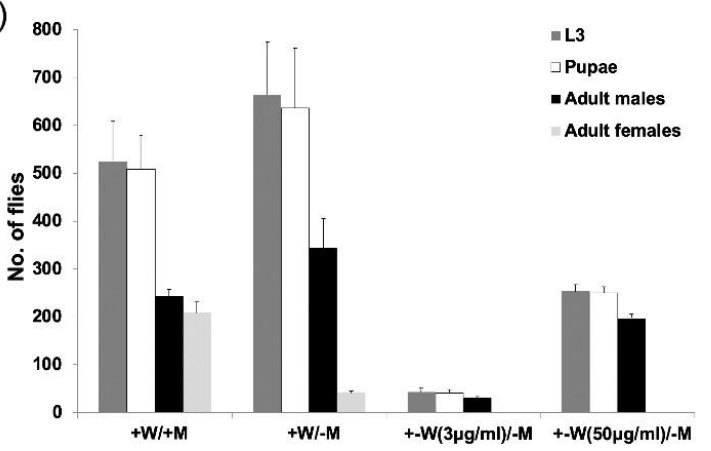

Figure 3. Female-specific lethality of TESS. The DR2\#7; EF3A (a) and DR2\#8; EF3A (b) strains were raised under different tetracycline feeding regimens. Containers were set with 10 pairs of adults and the number of third instar larvae (L3), pupae and adult male and female offspring counted. +W: water with $100 \mu \mathrm{g} / \mathrm{mL}$ tetracycline from day 1 (D1) to D8; + -W: water with limited tetracycline at indicated concentration from D1 to D2, then switched to water without tetracycline from D3 to D8; +M: ground meat (larval diet) with $100 \mu \mathrm{g} / \mathrm{g}$ tetracycline; $-\mathrm{M}$ : meat without tetracycline. Mean and standard error are shown from three replicate experiments.

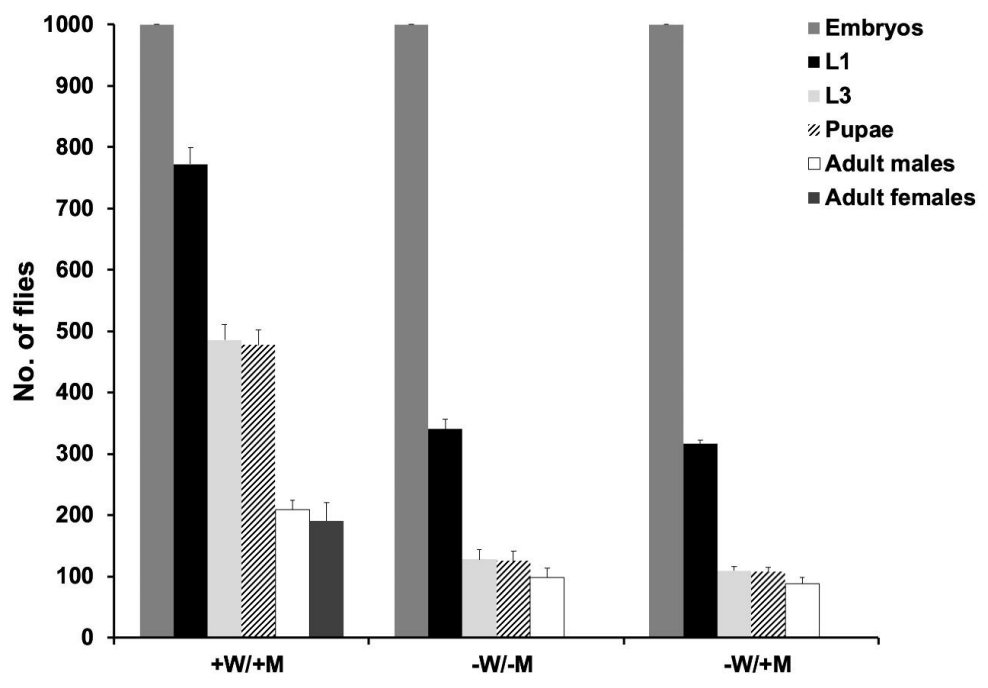

Figure 4. Staged lethality of the DR2\#7; EF3A strain. For each test, 1000 embryos were collected and the numbers of first instar larvae (L1), third instar larvae (L3), pupae, adult males and adult females were recorded. Error bars show the mean and standard error $(n=3)++\mathrm{W} /+\mathrm{M}$ : parental water contained $100 \mu \mathrm{g} / \mathrm{mL}$ tetracycline and the larval diet (93\% ground meat) contained $100 \mu \mathrm{g} / \mathrm{g}$ tetracycline; $-\mathrm{W} /-\mathrm{M}$ : neither adult nor larval diets contained tetracycline; $-\mathrm{W} /+\mathrm{M}$ : the adult diet lacked tetracycline but the larval diet contained $100 \mu \mathrm{g} / \mathrm{g}$ tetracycline. 


\section{Discussion}

The previously developed L. cuprina TESS strains with DR2 or DR3 drivers worked efficiently to eliminate females (100\% dominate lethality) at the embryo stage [16] or early larval stages [17]. However, the requirement for supplemental tetracycline to the adult diets of these TESS strains would add extra cost in labor and material for an SIT program, that involves mass-rearing of millions of insects. In addition, the maternally supplied tetracycline may partially suppress the lethal system in the release generation and lead to female survivors, resulting in a reduction in the program's control efficiency [24,25]. Moreover, reducing the usage of tetracycline could be beneficial since antibiotics eliminate some bacterial populations in insects with a consequent negative impact on fitness and male performance [26-28]. Out of the seven DH strains made in this study (Table 1, Figure S1), we successfully identified one strain DR2\#7; EF3A, which does not need an additional tetracycline supply for egg production but produces only male offspring. Therefore, this is an improvement over the previously reported TESS strains that used the DR2 driver as well but had undesired sterility. The male production of DR2\#7; EF3A is relatively low compared to some other TESS strains (Table 1), but could be compensated by seeding more eggs during mass rearing since females can be eliminated before the feeding stage.

The Lsrpr gene was less effective at inducing widespread cell death compared to Lshid when previously tested in Drosophila melanogaster using the GAL4-UAS system [22]. The A. suspensa rpr gene (Asrpr) also showed weaker proapoptotic activity than that of Ashid when tested in D. melanogaster S2 cells or the A. suspensa cell line UFENY-AsE01 [29]. Here we found an Lsrpr line in L. cuprina had no or very low female lethality when combined with DR2\#6 or DR3\#4 driver lines (Figure S1). In contrast, most of the DR2/DR3 and EF1/EF3 combinations tested previously were females lethal in the double heterozygous condition [16,17]. Thus, the results are consistent with our earlier observations that pro-apoptotic activity of Lsrpr was less than Lshid. However, it is possible that the low activity of the Lsrpr line is due to a negative position effect and thus additional transgenic lines would be required to confirm the low pro-apoptotic activity of Lsrpr in L. cuprina. While Lsrpr was relatively ineffective, some other strategies could be considered to build a moderate effector. For example, the EF3 construct comprised 21 copies of the tetO and the $h s p 70$ core promoter from L. cuprina $\left(\operatorname{tet} \mathrm{O}_{21^{-}}\right.$ Lchsp70-Lshid) [16], and previous studies showed that the seven copies of the tetO could also be used for embryonic lethality $[14,15,30]$ and the Dmhsp 70 core promoter mediated weaker effector gene activity compared to Lchsp70 core promoter in L. cuprina transgenic sexing strains [31]. Thus, by reducing the number of tetO copies in the effector and using a less active core promoter the expression of Lshid could be less responsive to tTA. Furthermore, since multiple copies of pro-apoptotic genes cause more intensive apoptosis than a single gene [29], it is possible to build a moderate effector with several copies of gene(s) showing weak pro-apoptotic activity that are co-expressed using the picornaviral self-cleaving 2A peptides [32]. Lastly, a moderate driver or effector can be generated by site-specific integration to a genomic site with known position effects on the transgene expression using either recombinase-mediated cassette exchange [33] or CRISPR/Cas9-mediated gene knock-in [34,35]. For example, a driver or effector cassette with strong expression/activity can be inserted into a genomic site with known negative position effects.

The lethality tests from different DH strains made in this study (Table 1) provide some practical information for the future development of functional TESS. First, an effector line with apparent low pro-apoptotic activity (EF3F or EF4) does not result in sufficient female lethality even when combined with a strong driver line (DR2\#8). Second, TESS females are sensitive to the level of tTA expression since females from DR2\#7; EF3A were fertile but DR2\#8; EF3A females were completely sterile if the adult diet lacked tetracycline. Third, the results from the DR2\#8; EF1-13 strain showed that an effective TESS can be made by combining a driver with high levels of tTA expression with a strong effector line but only if the adult diet is supplemented with a high dose of tetracycline for the first two days after eclosion. Fourth, crosses with a tetO-fluorescent protein reporter line can be used to identify tTA driver lines that have high expression in embryos but no expression in ovaries. Indeed, we recently 
found that driver lines made with the early promoters from the L. cuprina nullo or Cochliomyia macellaria CG14427 genes show high activity in embryos but no detectable $t$ TA expression in female ovaries [21]. TESS assembled with either of these two drivers had high female fertility on adult diet without tetracycline and 100\% female embryo lethality [21]. However, the identification and evaluation of an ideal promoter or pro-apoptotic gene for TESS development are largely dependent on the availability of genome resources and an efficient in vivo expression system, both of which could be very limited for some insect species. The Lsbnk promoter used in this study to drive tTA expression was obtained from L. sericata by a PCR-based genome walking approach [22]. With the availability of whole genome sequences for L. cuprina [36] and C. hominivorax [37], future driver strains can be made with gene promoters isolated from the species of interest.

\section{Conclusions}

Here we generated and evaluated several TESS strains for a potential genetic control program for L. cuprina. We showed one TESS strain, DR2\#7; EF3A, that was an improvement over previous TESS strains that had undesired female sterility. This strain does not need supplemental tetracycline for egg production and eliminates all female offspring at the embryo stage. Therefore, DR2\#7; EF3A is representative of the type of functional TESS strain that could lead to considerable savings in the running costs of an SIT program due to early and efficient female lethality and the generation of a male-only population for release.

\section{Data Availability}

The NCBI accession number for EF4 is MW150993. The DR2, EF1 and EF3 plasmids were previously published [16] with accession numbers KT749916, KT749917 and KT749918 respectively.

Supplementary Materials: The following are available online at http:/www.mdpi.com/2075-4450/11/11/797/s1, Figure S1. Female viability of TESSs with a L. sericata reaper effector gene. (a), the EF4 gene construct contains a marker cassette ( $L$ chsp83 promoter-RFPex-Lctub polyA) and a sex-specific effector cassette (tetO ${ }_{21}-L c h s p 70$ core promoter-Chtra female-specific intron-Lsrpr-SV40 polyA) flanked by the ends of the piggyBac transposon. (b), male and female viability of the DR2\#6;EF4 and DR3\#4;EF4 DH strains raised on diet without tetracycline. Eggs collected from 10 pairs of adults from each DH strain and the insects reared on $93 \%$ beef. The mean number of third instar larvae (L3), pupae and adult male and female with standard error are shown $(n=3)$. In the previous study, seven independent EF1 lines were obtained and all of them achieved $100 \%$ female lethality when crossed with DR2\#6 at double heterozygous condition, and seven independent EF3 lines were obtained and six of them achieved $96-100 \%$ female lethality (the remaining one showed $66 \%$ female lethality) when tested under the same condition [16]. In addition, DR3\#4 also achieved high female lethality when tested with several EF1/EF3 lines at double heterozygous condition, and relatively high $t T A$ expression was identified in the ovaries from DR3\#4 [17]. Here DR2\#6;EF4 showed no female lethality at double homozygous condition, indicating less pro-apoptotic activity of Lsrpr compared to that of Lshid in L. cuprina. On the other hand, $64 \%$ female lethality was detected from DR3\#4;EF4 DH strain, possibly due to the relatively high tTA expression at late developmental stages from DR3\#4 (Figure 1), suggesting the killing efficiency of Lsrpr effector also depends on tTA expression from the driver. Therefore, both the expression of the driver and effector are important for female lethality in the absence of tetracycline.

Author Contributions: Y.Y.: Conceptualization, Formal analysis, Investigation, Methodology, Project administration, Validation, Visualization, Writing—original draft. M.E.W.: Investigation, Methodology, Validation. M.J.S.: Funding acquisition, Project administration, Resources, Conceptualization, Supervision, Validation, Visualization, Writing - review and editing. All authors have read and agreed to the published version of the manuscript.

Funding: This research was funded by specific cooperative agreements between the USDA-ARS and NCSU and a grant from the Panama-United States Commission for the Eradication and Prevention of Screwworm (COPEG).

Acknowledgments: We thank Amy Keeter, Jodie White and Mary Hester for assistance with fly rearing, and Rebecca J. Davis for the help in qPCR experiments. We are grateful to Esther J. Belikoff for her comments and suggestions on the manuscript. The project benefitted from discussions at International Atomic Energy Agency funded meetings for the Coordinated Research Project: "The Use of Molecular Tools to Improve the Effectiveness of SIT" and "Comparing Rearing Efficiency and Competitiveness of Sterile Male Strains Produced by Genetic, Transgenic or Symbiont-based Technologies". The authors acknowledge the use of the Cellular and Molecular Imaging Facility (CMIF) at North Carolina State University, which is supported by the State of North Carolina and the National Science Foundation. Funding is gratefully acknowledged from specific cooperative agreements 
between the USDA-ARS and NCSU and a grant from the Panama-United States Commission for the Eradication and Prevention of Screwworm (COPEG).

Conflicts of Interest: The authors declare no conflict of interest.

\section{References}

1. Heath, A.C.; Bishop, D.M. Flystrike in New Zealand: An overview based on a 16-year study, following the introduction and dispersal of the Australian sheep blowfly, Lucilia cuprina Wiedemann (Diptera: Calliphoridae). Vet. Parasitol. 2006, 137, 333-344. [CrossRef]

2. Smith, R. Battling the blowfly. In Beyond the Bale; Australian Wool Innovation: Sydney, NSW, Australia, 2007; p. 2.

3. Williams, K.; Villet, M.H. Ancient and modern hybridization between Lucilia sericata and L. cuprina (Diptera: Calliphoridae). Eur. J. Entomol. 2013, 110, 187-196. [CrossRef]

4. Owings, C.G.; Picard, C.J. New Distribution Record for Lucilia cuprina (Diptera: Calliphoridae) in Indiana, United States. J. Insect Sci. 2018, 18, 1-6. [CrossRef]

5. Heath, A.C.G.; Bishop, D.M. Flystrike in New Zealand. Surveillance 1995, 22, 11-13.

6. Tellam, R.L.; Bowles, V.M. Control of blowfly strike in sheep: Current strategies and future prospects. Int. J. Parasitol. 1997, 27, 261-273. [CrossRef]

7. Scott, M.J.; Concha, J.B.; Welch, P.L.; Skoda, S.R. Research Advances in the Screwworm Eradication Program Over the Past 25 Years. Entomol. Exp. Appl. 2017, 164, 226-236. [CrossRef]

8. Foster, G.G.; Weller, G.L.; James, W.J.; Paschalidis, K.M.; McKenzie, L.J. Advances in sheep blowfly genetic control in Australia. In Management of Insect Pests: Nuclear and Related Molecular and Genetic Techniques; IAEA, Ed.; International Atomic Energy Agency: Vienna, Austria, 1993; pp. 299-312.

9. Knipling, E.F. Sterile-male method of population control. Science 1959, 130, 902-904. [CrossRef]

10. Foster, G.G. Chromosomal inversions and genetic control revisited: The use of inversions in sexing systems for higher Diptera. Theor. Appl. Genet. 1991, 81, 619-623. [CrossRef]

11. Rendon, P.; McInnis, D.; Lance, D.; Stewart, J. Medfly (Diptera: Tephritidae) genetic sexing: Large-scale field comparison of males-only and bisexual sterile fly releases in Guatemala. J. Econ. Entomol. 2004, 97, 1547-1553. [CrossRef]

12. Heinrich, J.C.; Scott, M.J. A repressible female-specific lethal genetic system for making transgenic insect strains suitable for a sterile-release program. Proc. Natl. Acad. Sci. USA 2000, 97, 8229-8232. [CrossRef] [PubMed]

13. Thomas, D.D.; Donnelly, C.A.; Wood, R.J.; Alphey, L.S. Insect population control using a dominant, repressible, lethal genetic system. Science 2000, 287, 2474-2476. [CrossRef] [PubMed]

14. Schetelig, M.F.; Handler, A.M. A transgenic embryonic sexing system for Anastrepha suspensa (Diptera: Tephritidae). Insect Biochem. Mol. Biol. 2012, 42, 790-795. [CrossRef]

15. Ogaugwu, C.E.; Schetelig, M.F.; Wimmer, E.A. Transgenic sexing system for Ceratitis capitata (Diptera: Tephritidae) based on female-specific embryonic lethality. Insect Biochem. Mol. Biol. 2013, 43, 1-8. [CrossRef]

16. Yan, Y.; Scott, M.J. A transgenic embryonic sexing system for the Australian sheep blow fly Lucilia cuprina. Sci. Rep. 2015, 5, 16090. [CrossRef] [PubMed]

17. Yan, Y.; Linger, R.J.; Scott, M.J. Building early-larval sexing systems for genetic control of the Australian sheep blow fly Lucilia cuprina using two constitutive promoters. Sci. Rep. 2017, 7, 2538. [CrossRef]

18. Li, F.; Vensko, S.P.I.; Belikoff, E.J.; Scott, M.J. Conservation and sex-specific splicing of the transformer gene in the Calliphorids Cochliomyia hominivorax, Cochliomyia macellaria and Lucilia sericata. PLoS ONE 2013, 8, e56303. [CrossRef]

19. Atkinson, P.W.; Pinkerton, A.C.; O’Brochta, D.A. Genetic transformation systems in insects. Annu. Rev. Entomol. 2001, 46, 317-346. [CrossRef]

20. Schetelig, M.F.; Targovska, A.; Meza, J.S.; Bourtzis, K.; Handler, A.M. Tetracycline-suppressible female lethality and sterility in the Mexican fruit fly, Anastrepha ludens. Insect Mol. Biol. 2016, 25, 500-508. [CrossRef]

21. Yan, Y.; Williamson, M.E.; Davis, R.J.; Andere, A.A.; Picard, C.J.; Scott, M.J. Improved transgenic sexing strains for genetic control of the Australian sheep blow fly Lucilia cuprina using embryo-specific gene promoters. Mol. Genet. Genom. 2020, 295, 287-298. [CrossRef] 
22. Edman, R.M.; Linger, R.J.; Belikoff, E.J.; Li, F.; Sze, S.H.; Tarone, A.M.; Scott, M.J. Functional characterization of calliphorid cell death genes and cellularization gene promoters for controlling gene expression and cell viability in early embryos. Insect Mol. Biol. 2015, 24, 58-70. [CrossRef]

23. Bergmann, A.; Agapite, J.; McCall, K.; Steller, H. The Drosophila gene hid is a direct molecular target of Ras-dependent survival signaling. Cell 1998, 95, 331-341. [CrossRef]

24. Franz, G. Genetic Sexing Strains in Mediterranean Fruit Fly, an Example for Other Species Amenable to Large-Scale Rearing for the Sterile Insect Technique. In Sterile Insect Technique: Principles and Practice in Area-Wide Integrated Pest Management; Dyck, V.A., Hendrichs, J., Robinson, A., Eds.; Springer: Dordrecht, The Netherlands, 2005.

25. Papathanos, P.A.; Bossin, H.C.; Benedict, M.Q.; Catteruccia, F.; Malcolm, C.A.; Alphey, L.; Crisanti, A. Sex separation strategies: Past experience and new approaches. Malaria J. 2009, 8, S5. [CrossRef]

26. Koga, R.; Tsuchida, T.; Sakurai, M.; Fukatsu, T. Selective elimination of aphid endosymbionts: Effects of antibiotic dose and host genotype, and fitness consequences. FEMS Microbiol. Ecol. 2007, 60, 229-239. [CrossRef]

27. Zhong, J.; Jasinskas, A.; Barbour, A.G. Antibotic treatment of the tick vector Amblyomma americanum reduced reproductive fitness. PLoS ONE 2007, 2, e405. [CrossRef]

28. Yuval, B.; Ben-Ami, E.; Behar, A.; Ben-Yosef, M.; Jurkevitch, E. The Mediterranean fruit fly and its bacteria-potential for improving sterile insect technique operations. J. Appl. Entomol. 2013, 1, $39-42$. [CrossRef]

29. Schetelig, M.F.; Nirmala, X.; Handler, A.M. Pro-apoptotic cell death genes, hid and reaper, from the tephritid pest species, Anastrepha suspensa. Apoptosis 2011, 16, 759-768. [CrossRef]

30. Horn, C.; Wimmer, E.A. A transgene-based, embryo-specific lethality system for insect pest management. Nat. Biotechnol. 2003, 21, 64-70. [CrossRef]

31. Li, F.; Wantuch, H.A.; Linger, R.J.; Belikoff, E.J.; Scott, M.J. Transgenic sexing system for genetic control of the Australian sheep blow fly Lucilia cuprina. Insect Biochem. Mol. Biol. 2014, 51, 80-88. [CrossRef]

32. Schwirz, J.; Yan, Y.; Franta, Z.; Schetelig, M.F. Bicistronic expression and differential localization of proteins in insect cells and Drosophila suzukii using picornaviral 2A peptides. Insect Biochem. Mol. Biol. 2020, 119, 103324. [CrossRef]

33. Schetelig, M.F.; Yan, Y.; Zhao, Y.; Handle, A.M. Genomic targeting by recombinase-mediated cassette exchange in the spotted wing drosophila, Drosophila suzukii. Insect Mol. Biol. 2018, 1-9. [CrossRef]

34. Davis, R.J.; Belikoff, E.J.; Scholl, E.H.; Li, F.; Scott, M.J. no blokes is essential for male viability and X chromosome gene expression in the Australian sheep blowfly. Curr. Biol. 2018, 28, 1987-1992.e1983. [CrossRef]

35. Paulo, D.F.; Williamson, M.E.; Arp, A.P.; Li, F.; Sagel, A.; Skoda, S.R.; Sanchez-Gallego, J.; Vasquez, M.; Quintero, G.; de Leon, A.A.P.; et al. Specific gene disruption in the major livestock pests Cochliomyia hominivorax and Lucilia cuprina Using CRISPR/Cas9. G3 2019, 9, 3045-3055. [CrossRef]

36. Anstead, C.A.; Korhonen, P.K.; Young, N.D.; Hall, R.S.; Jex, A.R.; Murali, S.C.; Hughes, D.S.; Lee, S.F.; Perry, T.; Stroehlein, A.J.; et al. Lucilia cuprina genome unlocks parasitic fly biology to underpin future interventions. Nat. Commun. 2015, 6, 7344. [CrossRef]

37. Scott, M.J.; Benoit, J.B.; Davis, R.J.; Bailey, S.T.; Varga, V.; Martinson, E.O.; Hickner, P.V.; Syed, Z.; Cardoso, G.A.; Torres, T.T.; et al. Genomic analyses of a livestock pest, the New World screwworm, find potential targets for genetic control programs. Commun. Biol. 2020, 3, 424. [CrossRef]

Publisher's Note: MDPI stays neutral with regard to jurisdictional claims in published maps and institutional affiliations.

(C) 2020 by the authors. Licensee MDPI, Basel, Switzerland. This article is an open access article distributed under the terms and conditions of the Creative Commons Attribution (CC BY) license (http://creativecommons.org/licenses/by/4.0/). 